\title{
EQUIVARIANT MINIMAX AND MINIMAL SURFACES IN GEOMETRIC THREE-MANIFOLDS
}

\author{
JON T. PITTS AND J. H. RUBINSTEIN
}

Minimal surfaces in Riemannian three-dimensional manifolds have played a major role in recent studies of the geometry and topology of 3-manifolds. In particular, stable and least area minimal surfaces have been used extensively [SY, MSY, HS]. On the other hand, explicit examples of unstable minimal surfaces have rarely been given. For the 3 -sphere $S^{3}$ with the standard metric of constant sectional curvature, there is the classical paper of Lawson [LH], showing that closed orientable surfaces of every genus occur as (unstable) minimal surfaces embedded in $S^{3}$. More recently, Karcher, Pinkall, and Sterling [KPS] have constructed several new examples in $S^{3}$.

We have discovered new infinite families of minimal surfaces in $S^{3}$. More generally, we announce a number of new finite and infinite families of embedded minimal surfaces in geometric 3-manifolds, using the minimax procedure described below. Geometric structures on 3-manifolds were introduced by Thurston [TW]. (See also the excellent survey by Scott [SP1].) There are eight geometries: $\mathbf{R}^{3}, S^{3}, S^{2} \times \mathbf{R}, \mathrm{Nil}, \mathbf{H}^{2} \times \mathbf{R}, \mathrm{SL} \widetilde{(2, \mathbf{R})}$, Solv, and $\mathbf{H}^{3}$. A geometric structure on a 3-manifold $\Sigma$ is a representation of $\Sigma$ as a quotient of one of the above eight spaces divided out by a covering transformation group acting isometrically. Equivalently, $\Sigma$ is locally isometric to one of these spaces, with its natural homogeneous space structure. The first six of these geometries give Seifert fiber spaces and we are mainly interested in such examples. The SO(2)-isometry actions associated with most Seifert fiber spaces yield infinite classes of embedded minimal surfaces. Note that interesting examples can also be obtained in the other two geometries; e.g., in hyperbolic geometry $\mathbf{H}^{3}$ [PR3, §2].

The minimax procedure has proved to be a versatile and powerful means of constructing unstable minimal surfaces in 3-manifolds. For basic details of this technique, see [PJ, SS, PR1, and PR2]. Suppose that $G$ is a finite group of isometries acting on a closed oriented Riemannian 3-manifold $\Sigma$. Assume that $\Lambda$ is a Heegaard surface in $\Sigma$; i.e., the closures of the components of $\Sigma \sim \Lambda$ are handlebodies $K$ and $K^{\prime}$. Assume furthermore that $\Lambda$ is G-equivariant; i.e., $g \Lambda=\Lambda$ for all $g \in G$. We consider one-parameter smooth families $\Lambda_{t}$, $t \in[0,1]$, sweeping out $\Sigma$ and having the following properties: $\Lambda_{0}$ and $\Lambda_{1}$ are graphs; $\Lambda_{t}$ is isotopic to $\Lambda$ for all $0<t<1 ; \Lambda_{t}$ is $G$-equivariant for all $t$; the handlebody $K_{t}$ is chosen so that the orientation on $\Lambda_{t}$ is induced from that on

Received by the editors November 15, 1987.

1980 Mathematics Subject Classification (1985 Revision). Primary 49F22, 53A10.

Research of the first author supported in part by a grant from the National Science Foundation. 
$K_{t}$ coming from $\Sigma$; and $K_{t}$ converges to $\Lambda_{0}$ as $t \rightarrow 0+$ and to $\Lambda_{1}$ as $t \rightarrow 1-$. Application of the minimax method to such equivariant sweepouts yields the following basic theorem.

THEOREM. There are sequences of $G$-equivariant families $\Lambda_{t}^{i}$ and parameters $t_{i}$ such that as $i \rightarrow \infty, \Lambda_{t_{i}}^{i}$ converges (in the $\mathbf{F}$ metric for varifolds) to a closed, embedded, $G$-equivariant, minimal surface $M$ in $\Sigma$. $M$ satisfies $\operatorname{genus}(M) \leq \operatorname{genus}(\Lambda)$ and $\operatorname{index}_{G}(M) \leq 1 \leq \operatorname{index}_{G}(M)+\operatorname{nullity}_{G}(M)$.

REMARKS. (1) See [PR2] for the definition of the F-metric. Index $\operatorname{din}_{G}(M)$ and nullity $_{G}(M)$ refer here to the $G$-equivariant index and nullity of $M$; i.e., the number of negative (respectively zero) eigenvalues of the second variation operator on $G$-equivariant normal vector fields to $M$, counted with multiplicity.

(2) We have stated this theorem in the context of sweepouts which depend on only one parameter. There is also a version which is valid for sweepouts with multiple parameters.

(3) $M$ is $G$-equivariant, but may be nonconnected or even nonorientable. We define

$$
\operatorname{genus}(M)=\sum_{j} n_{j} \operatorname{genus}\left(M_{j}\right)+\sum_{k} \frac{n_{k}}{2} \operatorname{genus}\left(M_{k}\right),
$$

where $M_{j}$ (respectively $M_{k}$ ) is an orientable (respectively nonorientable) component of $M$ with multiplicity $n_{j}$ (respectively $n_{k}$ ), where multiplicity of the components is as varifolds.

(4) $M$ is obtained from $\Lambda$ by deformations which may include both compressions of $\Lambda$ and projections of some components of the resulting surface onto double covers of nonorientable pieces $M_{k}$. A compression $\Lambda^{\prime}$ of $\Lambda$ is obtained by taking $\Lambda^{\prime}=(\Lambda \cup \partial N(D)) \sim \partial N(\partial D)$, where $D$ is an embedded disk in $\Sigma$ with $D \cup \Lambda=\partial D$, and $N(D)$ is a small product neighborhood of $D$, chosen so that $N(D) \cap \Lambda$ is an annular neighborhood $N(\partial D)$ of $\partial D$.

1. The 3-sphere. We list infinite classes of new examples. Our principal results are summarized in Table 1. We consider 14 different families of discrete subgroups of $\mathrm{SO}(4)$ regarded as symmetry groups of $S^{3}$ (column 1). Each family contains an infinite number of symmetry groups $G$ parameterized by one or more positive integers $m, k, r, s$. For each such group $G$, we obtain an embedded $G$-equivariant minimal surface in $S^{3}$, whose genus we calculate (column 2). Finally, within each family we study the geometry of the minimal surfaces obtained by analyzing limiting behavior of these surfaces as the order of the group $G$ increases without bound (column 3). More precisely, within each family, we have fixed parameters $k, r, s$ (if they occur), and have constructed a sequence $\left\{M_{m}\right\}$ of $G-(=G(m)-)$ equivariant minimal surfaces in $S^{3}$ which converges to the varifold $V$ described in column 3 as $m \rightarrow \infty$.

REMARKS. (1) Using other examples of finite groups acting on $S^{3}$, we are also able to construct minimal surfaces with the same genus and symmetry groups as those of Karcher, Pinkall, and Sterling [KPS], plus a few new examples of the same type not considered here. 


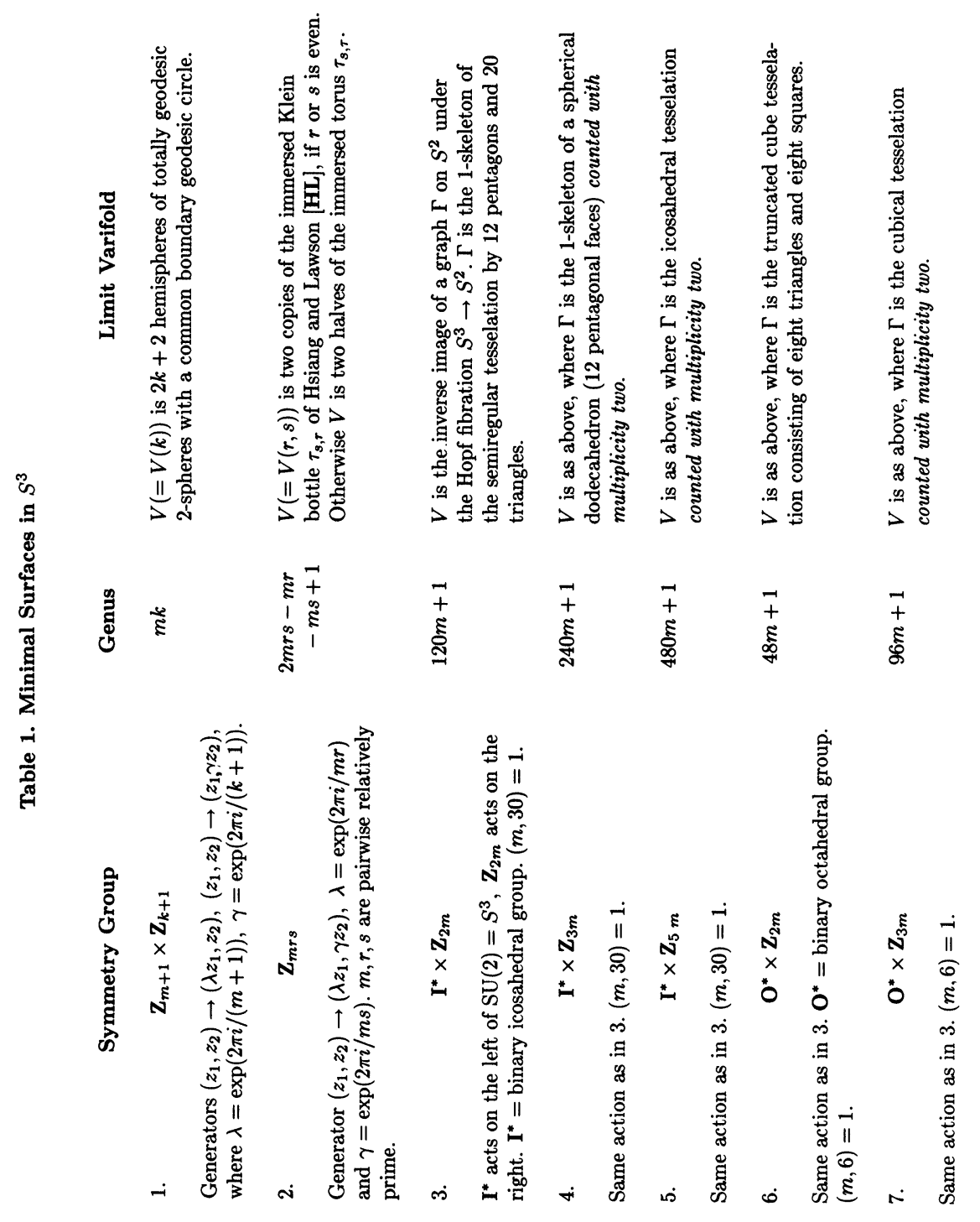




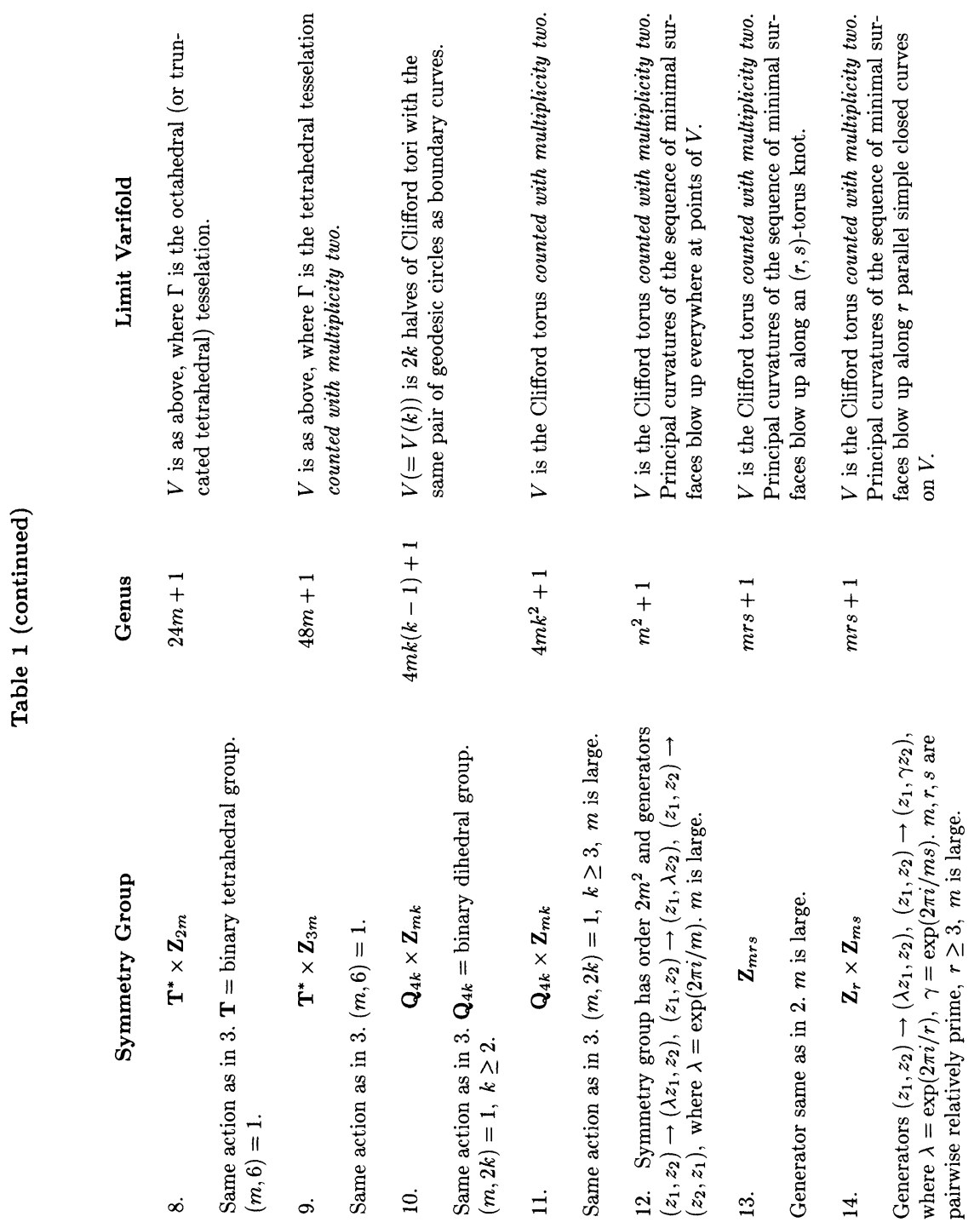


(2) The list of minimal surfaces in Table 1 is not exhaustive. The important case of free actions on $S^{3}$ is handled by the minimax procedure in [PR2]. In addition, for a given symmetry group, one can often obtain new surfaces from the minimax construction by considering more complicated sweepouts with multiple parameters. Finally, H. Karcher and we have recently discovered another fairly explicit method by which a number of minimal surfaces in $S^{3}$ can also be constructed.

(3) A helpful way to picture the sweepouts is to pass to the quotient space $S^{3} / G$ under the action of the symmetry group $G$. In most cases, $S^{3} / G$ is either $S^{3}$ or a 3-dimensional lens space, and the surface $\Lambda_{t} / G$ is either a 2 sphere or a Heegaard torus, respectively. It is then easy to identity possible compressions and classify them. The genus is then conveniently computed by the Hurwitz formula for branched coverings of surfaces.

(4) Examples 11-14, where $V$ is a Clifford torus counted with multiplicity two, exhibit several remarkable properties. One can think of $V$ as being the limit of minimal surfaces which are almost two copies of the torus separated slightly and joined by an appropriate number of small tubes. In the limit, the tubes disappear altogether through compression. In all previous examples (say, our examples 1-10, or Lawson's surfaces $\xi_{m, k}$ as $m \rightarrow \infty$ ), the limit surface $V$ has a singular set (typically singular arcs) along which compression occurs. To our knowledge, this is the first example where both compression has occurred and the limit varifold is a regular surface.

One notes furthermore that these examples contradict the conjecture of Lawson that a minimal surface should divide $S^{3}$ into two regions of equal volume. A minimal surface contradicting this conjecture was constructed in [KPS]. Our examples exhibit a much stronger property; namely, it is clear that as $m$ becomes arbitrarily large, the volume of one of the regions becomes arbitrarily small. This is also true in examples 4, 5, 7, and 9.

Our proof of the existence of the surfaces in examples 11-14 depends on being able to construct explicitly appropriate sweepouts of $S^{3}$ for which the maximum area is strictly less than twice the area of the Clifford torus. This construction depends fundamentally on a new result which we call the catenoid estimate. It appears that this estimate will have many applications.

(5) Surfaces in example 1 of Table 1 have the same symmetry group and genus as the surfaces $\xi_{m, k}$ of Lawson [LH]. Presumably they are the same surfaces, although this does not seem to follow readily from the basic construction. It seems that it may be possible to recover Lawson's examples, however, by using more elaborate sweepouts.

(6) In examples 1, 2, and 10 of Table 1, we do not yet know that the limit varifolds $V$ are balanced; i.e., $V$ may meet a singular circle in half-sheets and thus may not be an immersion. In contrast, one readily verifies that a sequence $\xi_{m, k}$ of Lawson surfaces converges as $m \rightarrow \infty$ to a balanced varifold $V$ which is the union of $k+1$ totally geodesic 2 -spheres intersecting in a common geodesic circle. We expect that $V$ is always balanced.

2. Other geometries. Here we give four methods for constructing minimal surfaces on manifolds with other geometries. 
FIRST CONSTRUCTION. Let $\Sigma$ be a $(2,3,7)$ Seifert fiber space; i.e., $\Sigma$ has three exceptional fibers of multiplicity two, three, and seven. (See Orlik [OP] for details on terminology.) Then $\Sigma$ has either the $\mathbf{H}^{2} \times \mathbf{R}$ or SL $\widetilde{(2, \mathbf{R})}$ geometry, depending on whether or not the Euler class of an appropriate $S^{1}$ bundle cover is zero. One takes as a symmetry group $G$ a finite cyclic subgroup of the $\mathrm{SO}(2)$ action along the Seifert fibers.

(a) $G=\mathbf{Z}_{7 m}$. The minimal surface $M$ has genus $6 m+1$. As $m \rightarrow \infty$, the limit varifold $V$ is twice a varifold $W$ which can be viewed conveniently by constructing its induced cover $\tilde{W}$ in the universal cover of $\Sigma$, which is either $\mathbf{H}^{2} \times \mathbf{R}$ or SL $\left.\widetilde{(2,} \mathbf{R}\right)$. To obtain $\tilde{W}$, one forms the tesselation of $\mathbf{H}^{2}$ by hyperbolic equilateral triangles with all angles $2 \pi / 7$, and multiplies the 1-skeleton $\Gamma$ of this triangulation by $\mathbf{R}$ (in the $\mathbf{H}^{2} \times \mathbf{R}$ case) or pulls back $\Gamma$ to $\operatorname{SL} \widetilde{(2, R)}$ under the natural projection $\operatorname{SL} \widetilde{(2, R)} \rightarrow \mathbf{H}^{2}$ (in the $\operatorname{SL} \widetilde{(2, R)}$ case).

(b) $G=\mathbf{Z}_{3 m}$. One proceeds as in (a), except that the tesselation of $\mathbf{H}^{\mathbf{2}}$ is by regular hyperbolic heptagons with all angles $2 \pi / 3$.

(c) $G=\mathbf{Z}_{2 m}$. One proceeds as in (a), except that the tesselation is semiregular, using heptagons and triangles. Furthermore, the convergence of the minimal surfaces to $V$ is with multiplicity one; i.e., $V=W$.

SECOND CONSTRUCTION. In this case $G$ acts freely, so we can use a nonequivariant sweepout in the quotient manifold $\Sigma / G$. Assume $\Sigma$ has an $\mathbf{H}^{2} \times \mathbf{R}$ structure and is a $(p, q, r)$ Seifert fiber space, where $p, q, r \geq 4$. Let $G$ be a free $\mathbf{Z}_{m-1}$ action embedded in the natural $\mathrm{SO}(2)$ action on $\Sigma$. As $\Sigma / G$ has Heegaard genus two, there is a genus two embedded minimal surface in $\Sigma / G$ which one obtains from the minimax procedure [PR2]. This lifts to a genus $m$ surface in $\Sigma$. As $m \rightarrow \infty$, these surfaces converge to an $\mathrm{SO}(2)$ invariant immersed minimal torus $V$ in $\Sigma$.

In the orbifold $\Sigma / \mathrm{SO}(2), V / \mathrm{SO}(2)$ is a figure 8 geodesic of shortest length, as described by Scott [SP2]. In the universal cover $\mathbf{H}^{2} \times \mathbf{R}$ of $\Sigma$, the induced cover $\tilde{V}$ is $\Gamma \times \mathbf{R}$, where $\Gamma$ is the 1-skeleton of the dual tesselation on $\mathbf{H}^{2}$ to the tesselation by the hyperbolic quadrilateral with angles $\pi / p, 2 \pi / r, \pi / q, 2 \pi / r$, in that order. This explicit tesselation has $p$-gons, $q$-gons, and $2 r$-gons. One notes that the choice of which of $p, q, r$ occurs with the factor 2 is determined by which of the three figure 8 geodesics is shortest.

If $p, q, r$ are small $(\leq 3)$, the situation is similar but more complicated (see Scott [SP2]).

THIRD CONSTRUCTION. Triply periodic minimal surfaces in $\mathbf{R}^{3}$ can be constructed which converge to the following varifolds $V$. In all cases $V$ is the 1-skeleton $\Gamma$ of a tesselation on $\mathbf{R}^{2}$ multiplied by $\mathbf{R}$. Here $\Sigma$ is the 3-torus, and $G=\mathbf{Z}_{r} \times \mathbf{Z}_{s}$ where $r=3, s=3 p$, or $r=4, s=2 p$, or $r=6, s=2 p$ or $3 p$, and $p$ is relatively prime to 6 . These are the possibilities:

(a) $\Gamma$ is the equilateral triangle tesselation.

(b) $\Gamma$ is the square tesselation (and $M$ is analogous to Scherk's periodic surface).

(c) $\Gamma$ is the semiregular tiling by hexagons and equilateral triangles.

(d) $\Gamma$ is the tiling by hexagon with multiplicity two. 
FOURTh CONSTRUCTION. In $S^{2} \times S^{1}$, minimal surfaces can be found which converge to varifolds $V$ of the form $\Gamma \times S^{1}$, where $\Gamma$ is the 1-skeleton of a tesselation of $S^{2}$. Finally, Nil geometry has various $S^{1}$-bundles over the 2torus. Once again examples can be constructed which converge to $S^{1}$-bundles over the 1-skeleton of tesselations of the 2-torus, as in the third construction above.

\section{REFERENCES}

[HS] J. Hass and P. Scott, The existence of least area surfaces, preprint.

[HL] W. Hsiang and H. B. Lawson, Minimal submanifolds of low cohomogeneity, J. Differential Geom. 5 (1971), 1-38.

[KPS] H. Karcher, U. Pinkall, and I. Sterling, New minimal surfaces in $S^{3}$, preprint.

[LH] H. B. Lawson, Complete minimal surfaces in $S^{3}$, Ann. of Math. (2) 90 (1970), 335-374.

[MSY] W. Meeks, L. Simon, and S. T. Yau, Embedded minimal surfaces, exotic spheres, and manifolds with positive Ricci curvature, Ann. of Math. (2) 116 (1982), 621-659.

[OP] P. Orlik, Seifert fiber spaces, Lecture Notes in Math., vol. 291, Springer-Verlag, Berlin and New York, 1981.

[PJ] J. Pitts, Existence and regularity of minimal surfaces on Riemannian manifolds, Math. Notes 27, Princeton Univ. Press, Princeton, N. J., 1981.

[PR1] J. Pitts and J. H. Rubinstein, Existence of minimal surfaces of bounded topological type in three-manifolds, Proceedings of the Centre for Mathematical Analysis 10 (1986), 163-176.

[PR2] _ _ Minimal surfaces of bounded topological type in three-manifolds, preprint.

[PR3] _ Applications of minimax to minimal surfaces and the topology of 3-manifolds, Proc. Centre Math. Anal. 12 (1987), 137-170.

[SY] R. Schoen and S. T. Yau, Existence of incompressible minimal surfaces and the topology of three dimensional manifolds with nonnegative scalar curvature, Ann. of Math. (2) 110 (1979), 127-145. 487.

[SP1] P. Scott, The geometries of 3-manifolds, Bull. London Math. Soc. 15 (1983), 401(1983), 35-70.

[SS] L. Simon and F. Smith, On the existence of embedded minimal 2-spheres in the 3sphere, endowed with an arbitrary metric, preprint.

[TW] W. Thurston, Geometry and topology of 3-manifolds, mimeographed lecture notes, Princeton Univ., 1978.

Department of Mathematics, Texas A \& M University, College StaTION, TEXAS 77843

Department of Mathematics, University of Melbourne, Parkville, Victoria, AUstralia 
